\title{
O impacto de um programa social brasileiro sobre a saúde bucal de crianças
}

\author{
The impact of a Brazilian social program on children's oral health
}

\author{
Valdeci Elias dos Santos Junior \\ Arnoldo Vasconselos de Alencar Filho ${ }^{* *}$ \\ Rebeca Maria Brasileiro de Sousa ${ }^{* * *}$ \\ Fábio Cavalcanti ${ }^{* * * *}$ \\ Monica Vilela Heimer ${ }^{* * * * *+}$ \\ Aronita Rosenblatt ${ }^{* * *+*}$
}

\section{Resumo}

Objetivo: este estudo avaliou o impacto do Programa Bolsa Família na prevalência de cárie dentária de crianças de 4 a 8 anos de idade que receberam esse benefício desde seu nascimento. Métodos: cento e sessenta e três crianças foram examinadas seguindo os critérios da Organização Mundial da Saúde para dentes cariados, perdidos e obturados. Resultados: verificou-se que a prevalência de cárie dentária das crianças incluídas no programa foi de $78,5 \%$ e que o índice de dentes cariados, extraídos e obturados (ceo-d) na dentição decídua médio foi de 4,77, com predominância do componente cariado, o qual representa $81,8 \%$ desse índice. O índice CPO-D (dentes cariados, perdidos e obturados na dentição permanente) encontrado foi de 0,56 , indicando um acometimento precoce de dentes permanentes recém-erupcionados. Conclusão: embora esse programa de transferência de renda possa promover alívio imediato da condição de extrema pobreza, as crianças nascidas e criadas obtendo esse benefício continuaram apresentando uma média de ceo-d quase o dobro da média regional verificada no último levantamento epidemiológico de saúde bucal realizado em 2010.

Palavras-chave: Criança. Cárie dentária. Programas governamentais.

\section{Introdução}

A pesquisa tem demonstrado que a condição socioeconômica é um importante indicador de risco em saúde e, consequentemente, da doença cárie ${ }^{1,2}$. $\mathrm{O}$ menor poder aquisitivo envolve um conjunto de fatores que dizem respeito ao acesso a serviços de saúde, nível educacional, estilo de vida, condições de higiene, moradia e acesso a produtos ${ }^{3}$. Dessa forma, grupos específicos da população permanecem com elevada prevalência de cárie dentária, uma vez que a vulnerabilidade ao agravo está associada à exposição mais intensa aos fatores de risco e à privação social ${ }^{4,5}$.

Com o intuito de diminuir o abismo social, a equidade enquanto discriminação positiva é um dos princípios fundamentais norteadores do Sistema Único de Saúde (SUS) no Brasil, de modo a garantir igualdade de assistência ${ }^{6}$. Assim, visando à redução e prevenção de danos sociais, surge o Programa Bolsa Família, criado em 9 de janeiro de 2004 pelo governo federal. O Bolsa Família integra o plano Brasil Sem Miséria (BSM), que tem como foco de

http://dx.doi.org/10.5335/rfo.v18i1.3019

Mestre e Doutor em Odontologia, área de concentração: Odontopediatria, pela Faculdade de Odontologia de Pernambuco, Universidade de Pernambuco, Recife, PE, Brasil.

Mestrando em Odontologia pela Universidade de Pernambuco, Faculdade de Odontologia de Pernambuco, Departamento de Odontologia Social, Recife, PE, Brasil.

*** Mestra em Odontologia, área de concentração: Odontopediatria, pela Faculdade de Odontologia de Pernambuco, Universidade de Pernambuco, Recife, PE, Brasil.

**** Professor Assistente da disciplina de Bioestatística da Universidade de Pernambuco, Faculdade de Odontologia de Pernambuco, Recife, $\mathrm{PE}$, Brasil.

***** Professora Doutora Adjunta da disciplina de Saúde Coletiva da Universidade de Pernambuco, Faculdade de Odontologia de Pernambuco, Departamento de Odontologia Social, Recife, PE, Brasil.

****** Professora Doutora Titular da Disciplina de Odontopediatria da Universidade de Pernambuco, Faculdade de Odontologia de Pernambuco, Departamento de Odontologia Social, Recife, PE, Brasil. 
atuação os 16 milhões de brasileiros com renda familiar per capita inferior a $\mathrm{R} \$ 70$ mensais e está baseado na garantia de renda, inclusão produtiva e no acesso aos serviços públicos. Consiste em um programa social de transferência direta de renda, que beneficia famílias em situação de pobreza e de extrema pobreza com base em três eixos principais: transferência de renda, condicionalidades e programas complementares ${ }^{7}$. A transferência de renda promove o alívio imediato da pobreza, e as condicionalidades reforçam o acesso a direitos sociais básicos nas áreas de educação, saúde e assistência social. Por sua vez, os programas complementares têm por objetivo o desenvolvimento das famílias, de modo que os beneficiários do Bolsa Família consigam superar a situação de vulnerabilidade e pobreza. São exemplos de programas complementares: programas de geração de trabalho e renda, de alfabetização de adultos, de fornecimento de registro civil e demais documentos ${ }^{8}$.

Todavia, os benefícios atuais promovidos pelo governo brasileiro derivados da concessão de dinheiro público devem ser alvo de pesquisas para a análise da real eficácia dos efeitos das condicionalidades resultantes do programa, a exemplo dos cuidados em saúde. Desse modo, faz-se necessário identificar indicadores que possam ter sido influenciados pelo contexto social, levando à ausência ou ao aparecimento de doenças, focado no impacto de programas assistenciais ${ }^{9}$.

Pelo exposto, o presente estudo buscou avaliar o impacto do Programa Bolsa Família na prevalência de cárie dentária de crianças de 4 a 8 anos de idade que receberam esse benefício desde seu nascimento. A hipótese defendida pelos autores é que crianças nascidas com acesso a esse benefício, dentro do BSM, apresentariam índice de saúde bucal médio próximo à média regional.

\section{Métodos}

Esta pesquisa foi realizada no município de Jaboatão dos Guararapes, Pernambuco, Nordeste do Brasil, que tem uma população de 644.620 habitantes ${ }^{10}$, dos quais 68.257 são beneficiários do Programa Bolsa Família, correspondendo a 10,5\% da população ${ }^{11}$. Esses beneficiários recebem do programa, em média, $\mathrm{R} \$ 1.128,33$ ao ano.

Este estudo foi aprovado pelo Comitê de Ética em Pesquisa da Universidade de Pernambuco e conduzido de acordo com os princípios éticos que concernem à Declaração de Helsinki.

O cálculo amostral foi realizado utilizando-se o programa Epiinfo $6^{\circledR}$ (CDC, Atlanta, Georgia, USA) baseado em estudo piloto desenvolvido em 30 crian- ças beneficiadas pelo Programa Bolsa Família, do qual se obteve uma prevalência de cárie de $88 \%$. Foram levados em consideração o erro tipo I de $5 \%$ e o intervalo de confiança de 95\%. Assim, estipulou-se o tamanho amostral de 163 crianças. A amostragem empregada foi do tipo aleatório simples, na qual uma lista concedida pela Secretaria de Assistência Social do município de Jaboatão dos Guararapes foi utilizada para a realização de sorteios dos cadastrados, de forma a dar a todos a mesma oportunidade de participar do estudo.

Todas as crianças foram examinadas na posição joelho a joelho, em um local com uma boa luminosidade em sua residência, utilizando o protocolo de biossegurança da Universidade de Pernambuco. No entanto, crianças com distúrbios motores e mentais foram excluídos da pesquisa para maior fidedignidade dos dados obtidos.

A cárie dentária foi avaliada por meio do ceo-d e CPO-D, índice de dentes cariados, perdidos e obturados, utilizado para a dentição decídua e permanente, respectivamente. Esses índices foram coletados mediante acesso às fichas clínicas, seguindo as orientações da Organização Mundial de Saúde $(\mathrm{OMS})^{9}$, com o objetivo de se estabelecer a prevalência e a severidade da cárie dentária.

Os exames foram conduzidos por quatro examinadores, previamente calibrados, por meio do teste de Kappa, recomendado pela OMS (1999) ${ }^{9}$. Os coeficientes Kappa intra e interexaminador para o diagnóstico de cárie foram, respectivamente, $\mathrm{K}=0,90 \mathrm{e}$ $\mathrm{K}=0,85$, indicando um bom nível de concordância.

Os dados desta pesquisa foram processados em banco de dados no programa Statistical Package for Social Science (SPSS ${ }^{\circledR}$ ) versão 15.0. Foi realizada uma análise descritiva dos dados dos componentes dos índices CPO-D e ceo-d e foram obtidas as médias e o desvio padrão. A estatística inferencial, por meio do teste de Mann-Whitney e qui-quadrado, foi aplicada para verificar se existia diferença estatisticamente significativa na distribuição desses índices (ceo-d e CPO-D) entre os sexos e faixa etária.

\section{Resultados}

Após a análise dos dados coletados, verificou-se que, das 163 crianças examinadas, 55,2\% (90) eram do sexo masculino e $44,8 \%$ (73) eram do sexo feminino, com idade média de 5,85, aproximadamente 6 anos.

Das crianças participantes, 78,5\% apresentavam pelo menos um dente cariado, não sendo observada diferença estatisticamente significante em relação ao sexo e à faixa etária (Tabela 1). 


\begin{tabular}{|c|c|c|c|c|c|c|c|c|}
\hline & & \multicolumn{7}{|c|}{ Dentes cariados } \\
\hline & & \multicolumn{2}{|c|}{ Sim } & \multicolumn{2}{|c|}{ Não } & \multicolumn{2}{|c|}{ Total } & \multirow[t]{2}{*}{$p$ - valor } \\
\hline & & $\mathrm{N}$ & $\%$ & $\mathrm{~N}$ & $\%$ & $\mathrm{n}$ & $\%$ & \\
\hline \multirow[b]{3}{*}{ Faixa etária } & Total & 128 & $78,5 \%$ & 35 & $21,5 \%$ & 163 & $100,0 \%$ & \\
\hline & 4 a 5 anos & 61 & $72,6 \%$ & 23 & $27,4 \%$ & 84 & $100,0 \%$ & $0_{1}$ \\
\hline & 6 a 8 anos & 67 & $84,8 \%$ & 12 & $15,2 \%$ & 79 & $100,0 \%$ & $\bigodot_{\infty}^{\infty}$ \\
\hline \multirow[b]{2}{*}{ Sexo } & Masculino & 71 & $78,9 \%$ & 19 & $21,1 \%$ & 90 & $100,0 \%$ & $\sigma_{\Xi}$ \\
\hline & Feminino & 57 & $78,1 \%$ & 16 & $21,9 \%$ & 73 & $100,0 \%$ & $\underset{0}{\overrightarrow{0}}$ \\
\hline
\end{tabular}

(*): Diferença significativa a 5,0\%

(1): Por meio do qui-quadrado de Pearson

Constatou-se que, das 163 crianças examinadas, 17,2\% apresentavam algum dente decíduo perdido e apenas 1,0\% possuía algum tipo de restauração (Tabela 2).

Tabela 2 - Média do índice ceo-d e proporção dos componentes do ceo-d, segundo a faixa etária

\begin{tabular}{|c|c|c|c|c|c|c|c|c|c|c|c|}
\hline \multirow{2}{*}{ Faixa etária } & \multirow{2}{*}{$\mathrm{N}$} & \multicolumn{2}{|c|}{ Cariado } & \multicolumn{2}{|c|}{ Obturado } & \multicolumn{2}{|c|}{ Perdido } & \multirow{2}{*}{$\begin{array}{l}\text { Ceo-d } \\
\text { Média }\end{array}$} & \multirow{2}{*}{ DP } & \multicolumn{2}{|c|}{ IC (95\%) } \\
\hline & & Média & $\%$ & Média & $\%$ & Média & $\%$ & & & L.I. & L.S. \\
\hline 4 a 5 anos & 84 & 3,42 & 85,2 & 0,02 & 0,6 & 0,57 & 14,2 & 4,01 & 3,89 & 3,18 & 4,84 \\
\hline 6 a 8 anos & 79 & 4,42 & 79,1 & 0,08 & 1,4 & 1,09 & 19,5 & 5,58 & 3,73 & 4,76 & 6,40 \\
\hline Total & 163 & 3,90 & 81,8 & 0,05 & 1,0 & 0,82 & 17,2 & 4,77 & 3,88 & 4,18 & 5,37 \\
\hline
\end{tabular}

O índice ceo-d para as idades de 4 a 5 anos foi de 4,01 e de 6 a 8 anos foi de 5,58 (Tabela 2). Nenhuma criança na faixa etária de 4 a 5 anos possuía dentes permanente cariados, corroborando um CPO-D igual a 0 para essa faixa etária (Tabela 3 ).

Tabela 3 - Média do índice CPO-D e proporção dos componentes do CPO-D, segundo a faixa etária

\begin{tabular}{|c|c|c|c|c|c|c|c|c|c|c|c|}
\hline \multirow{2}{*}{ Faixa etária } & \multirow{2}{*}{$N$} & \multicolumn{2}{|c|}{ Cariado } & \multicolumn{2}{|c|}{ Obturado } & \multicolumn{2}{|c|}{ Perdido } & \multirow{2}{*}{$\begin{array}{l}\text { CPO-D } \\
\text { Média }\end{array}$} & \multirow{2}{*}{ DP } & \multicolumn{2}{|c|}{ IC (95\%) } \\
\hline & & Média & $\%$ & Média & $\%$ & Média & $\%$ & & & L.I. & L.S. \\
\hline 4 a 5 anos & 5 & 0,00 & - & 0,00 & - & 0,00 & - & 0,00 & 0,00 & 0,00 & 0,00 \\
\hline 6 a 8 anos & 68 & 0,57 & 95,1 & 0,03 & 4,9 & 0,00 & 0,0 & 0,60 & 1,22 & 0,31 & 0,89 \\
\hline Total & 73 & 0,53 & 95,1 & 0,03 & 4,9 & 0,00 & 17,2 & 0,56 & 1,19 & 0,29 & 0,83 \\
\hline
\end{tabular}

Observou-se um CPO-D médio de 0,56 entre as 73 crianças que possuíam dentes permanentes (Tabela 3). Em relação à faixa etária, observou-se diferença significativa para o ceo-d $(p=0,003)$, o mesmo não sendo observado para o CPO-D $(\mathrm{p}=0,149)$ (Tabela 4$)$.

Tabela 4 - Análise descritiva do ceo-d e CPO-D por sexo e faixa etária

\begin{tabular}{|c|c|c|c|c|c|c|c|c|c|}
\hline Índice & Tipo & & $N$ & Mínimo & Máximo & Média & Mediana & DP & $p$ - Valor \\
\hline \multirow{6}{*}{ ceo-d } & \multirow{3}{*}{ Sexo } & Total & 163 & 0,00 & 16,00 & 4,77 & 4,00 & 3,88 & \multirow{3}{*}{ 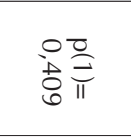 } \\
\hline & & Masculino & 90 & 0,00 & 16,00 & 4,60 & 4,00 & 3,93 & \\
\hline & & Feminino & 73 & 0,00 & 15,00 & 4,99 & 5,00 & 3,84 & \\
\hline & \multirow{3}{*}{ Faixa etária } & 4 a 5 anos & 84 & 0,00 & 16,00 & 4,01 & 3,50 & 3,89 & \multirow{3}{*}{ 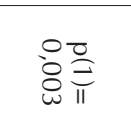 } \\
\hline & & 6 a 8 anos & 79 & 0,00 & 16,00 & 5,58 & 5,00 & 3,73 & \\
\hline & & Total & 73 & 0,00 & 6,00 & 0,56 & 0,00 & 1,19 & \\
\hline \multirow{4}{*}{ CPO-D } & \multirow{2}{*}{ Sexo } & Masculino & 35 & 0,00 & 6,00 & 0,74 & 0,00 & 1,36 & \multirow{2}{*}{ 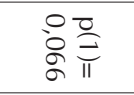 } \\
\hline & & Feminino & 38 & 0,00 & 4,00 & 0,39 & 0,00 & 1,00 & \\
\hline & \multirow{2}{*}{ Faixa etária } & 4 a 5 anos & 5 & 0,00 & 0,00 & 0,00 & 0,00 & 0,00 & \multirow{2}{*}{$\stackrel{\circ}{\stackrel{0}{\vec{b}}}$} \\
\hline & & 6 a 8 anos & 68 & 0,00 & 6,00 & 0,60 & 0,00 & 1,22 & \\
\hline
\end{tabular}

Diferença significativa a 5,0\%

(1): Por meio do teste Mann-Whitney 
Das 73 crianças participantes que possuíam dentes permanentes, 95,1\% apresentavam, ao menos, um desses dentes com cárie. Nenhuma perda dentária foi identificada, e apenas $4,9 \%$ tinham dentes restaurados (Tabela 3). Analisando-se apenas os portadores da doença, verificou-se uma média de 4,14 dentes cariados por criança (Tabela 5).

Tabela 5 - Média do índice ceo-d e CPO-D e proporção dos componentes do ceo-d e CPO-D total, segundo a faixa etária

\begin{tabular}{|c|c|c|c|c|c|c|c|c|c|c|c|c|c|}
\hline \multirow{3}{*}{ Faixa etária } & \multirow{3}{*}{$\mathrm{N}$} & \multirow{2}{*}{\multicolumn{2}{|c|}{ Cariado }} & \multirow{2}{*}{\multicolumn{2}{|c|}{ Obturado }} & \multirow{2}{*}{\multicolumn{2}{|c|}{ Perdido }} & \multirow{2}{*}{\multicolumn{3}{|c|}{$\begin{array}{c}\text { ceo-d } \\
\text { IC (95\%) }\end{array}$}} & \multirow{2}{*}{\multicolumn{3}{|c|}{$\begin{array}{l}\text { CPO-D } \\
\text { IC }(95 \%)\end{array}$}} \\
\hline & & & & & & & & & & & & & \\
\hline & & Média & $\%$ & & $\%$ & & $\%$ & & L.I. & L.S. & & L.I. & L.S. \\
\hline 4 a 5 anos & 84 & 3,42 & 85,2 & 0,02 & 0,6 & 0,57 & 14,2 & 4,01 & 3,18 & 4,84 & 0,00 & 0,00 & 0,00 \\
\hline 6 a 8 anos & 79 & 4,91 & 80,5 & 0,10 & 1,7 & 1,09 & 17,8 & 5,58 & 4,76 & 6,40 & 0,60 & 0,31 & 0,89 \\
\hline Total & 163 & 4,14 & 82,4 & 0,06 & 1,3 & 0,82 & 16,4 & 4,77 & 4,18 & 5,37 & 0,56 & 0,29 & 0,83 \\
\hline
\end{tabular}

\section{Discussão}

A causalidade social é inespecífica em contraste à capacidade da epidemiologia tradicional de vincular causas singulares com resultados singulares. Portanto, a filosofia cartesiana de característica positiva e concreta, demasiadamente simplória e prática, não se aplica ao processo saúde-doença, devido ao percurso não linear de fatores condicionantes e modificadores que o caracteriza. Partindo dessa premissa, a causalidade social não pode ser vista como fator de atuação uniforme, em razão da influência de um mundo repleto de disparidades sociais $^{12}$.

Contudo, como ilustram Mota e Carvalho ${ }^{13}$ (2003), a informação epidemiológica, quando fornecida com precisão, é de importância capital para a institucionalização de um modelo de vigilância à saúde como se deseja no SUS, pois tende a revelar perfis e tendências nas condições de saúde e reflexos na condição de evolução de uma sociedade, sendo, assim, aplicáveis a essas atividades.

O relatório do SB Brasil $2010^{9}$ mostrou que $54,3 \%$ das crianças de até 5 anos de idade apresentaram, em média, 2,43 dentes com cárie, com predomínio do componente cariado, que é responsável por mais de $80 \%$ do índice. No entanto, para o Nordeste, o ceo-d médio atingiu o valor de 2,89 , e o componente cariado correspondeu a 88,2\%. Em contrapartida, as crianças examinadas neste estudo, beneficiadas pelo Programa Bolsa Família desde o seu nascimento, apresentaram um ceo-d médio de 4,77 e o componente cariado correspondeu a $81,8 \%$ dessa média, de forma que a comparação desses dados epidemiológicos apontaram para a permanência de fortes iniquidades em saúde, uma vez que os resultados do presente estudo foram quase duas vezes $(1,95)$ superior ao resultado regional. Ainda analisando os resultados, embora o CPO-D seja de 0,56, deve-se notar que a faixa de idade das crianças envolvidas neste estudo foi de 5 a 8 anos de idade, o que indica que os elementos dentários eram recém-erupcionados e precocemente acometidos por cárie.
Considerando que esse índice é influenciado pelo contexto social, observa-se que essa discussão vai além dos limites de saúde bucal e adentra questões que norteiam as diretrizes da equidade social ${ }^{14}$. Experiências humanas podem alterar mecanismos biológicos estáveis e transformá-los em um legado genético, processo chamado de incorporação biológica $^{12}$. Nessa perspectiva, o impacto da inequidade social na condição de saúde bucal ao longo da vida foi alvo de investigação em 13 países europeus; para isso, 26.525 pessoas com idade média de 50 anos foram avaliadas. Os resultados desse estudo mostraram que as disparidades na condição de saúde bucal determinadas por iniquidades socioeconômicas, quando estabelecidas na infância, perpetuam-se até a fase adulta ${ }^{15}$.

Estudos realizados no continente africano ${ }^{16,17}$ mostraram uma menor prevalência de cárie na dentição decídua quando comparada com as crianças beneficiárias do Programa Bolsa Família. Vários fatores podem explicar essa diferença, entre os quais, o consumo de sacarose. Embora não tenha sido avaliado no presente estudo, sabe-se que no Brasil esse consumo é bem maior do que na África ${ }^{17}$. Além disso, a maior parte da verba transferida pelo Programa Bolsa Família é gasta com alimentação e, devido ao aumento do poder aquisitivo, cresceu o consumo de alimentos de alto poder cariogênico, como biscoitos e produtos industrializados ${ }^{19}$.

Segundos dados da Associação Brasileira da Indústria de Higiene Pessoal, Perfumaria e Cosméticos (ABIHPEC) ${ }^{20}$, as classes menos favorecidas consumiram $R \$ 1.700 .000,00$ em produtos de higiene pessoal no ano de 2010. Essa associação ainda relata que, nos últimos cinco anos, houve um aumento de $19 \%$ do consumo desses produtos pela referida classe social. No entanto, os reflexos da inserção desses itens ainda não foram, efetivamente, notados na melhoria da condição de saúde bucal da população estudada.

A OMS apresentou, como uma das metas de saúde bucal para o ano 2000 , que $50 \%$ das crianças com idade entre 5 e 6 anos estivessem livres de cárie. 
Para o ano de 2010, a meta foi ampliada para $90 \%$. Numa perspectiva menos ousada, a meta foi reduzida para $80 \%$ em $2020^{21}$. No entanto, no presente trabalho, observou-se que apenas $11 \%$ das crianças inseridas no Programa Bolsa Família estão livres da doença, condição bem distante das metas. Segundo o Ministério da Saúde, de 2003 a 2010, houve um aumento de $15,42 \%$ no número de crianças livres de cárie para essa faixa etária ${ }^{9}$, o que parece não ter ocorrido com o grupo de crianças analisadas neste estudo, resultado esperado, uma vez que a população alvo era uma amostra da camada menos favorecida dessa região do país, todos beneficiários de programa de redução de dano.

Vale salientar, ainda, que o componente obturado do ceo-d representa apenas $1 \%$ dos dentes examinados em toda a população estudada, o que demonstrou o quanto a doença cárie pode ser negligenciada, em algumas populações, por falta de acesso aos serviços públicos de saúde. Dessa forma, fica estabelecida a necessidade de se programar a atenção à saúde de maneira equânime, uma vez que o acesso à saúde distribui-se de forma não harmônica na população brasileira devido às variáveis sociais ${ }^{22}$. Logo, no intuito de diminuir o abismo social, a equidade enquanto discriminação positiva é um dos princípios fundamentais norteadores do SUS no Brasil, de modo a garantir igualdade de assistên$\operatorname{cia}^{7,9}$.

Segundo o Manual de orientação sobre o Programa Bolsa Família na saúde, os compromissos dos beneficiários responsáveis por crianças de até 7 anos de idade incluem: cumprir o calendário escolar e de vacinação da criança, participar de atividades educativas sobre aleitamento materno e cuidados gerais com a saúde da criança e levar a criança à unidade de saúde para o acompanhamento e desenvolvimento de acordo com o preconizado pelo Ministério da Saúde ${ }^{7}$. Porém, diferentemente dos dois primeiros compromissos, sobre os quais o governo exerce um controle social, a presença da criança na unidade de saúde não é acompanhada com o devido rigor. Ademais, os parâmetros de saúde bucal não são sequer mencionados para o controle social do programa. Dessa forma, a exemplo de outros países que dispõem de programas sociais, como a Colômbia, visitas regulares ao dentista deveriam fazer parte das condicionalidades obrigatórias do programa brasileiro, a fim de que esse controle propiciasse uma melhoria na condição de saúde bucal desses indivíduos.

Portanto, embora apresente resultados expressivos sobre a redução da desigualdade e da pobre$\mathrm{za}^{23}$, o Programa Bolsa Família ainda não exerce impactos positivos na condição de saúde bucal de seus beneficiários, ainda que estes tenham sido contemplados desde sua mais tenra idade.

\section{Conclusão}

Embora contempladas desde o nascimento pelo Programa Bolsa Família, as crianças que participaram desta pesquisa, pertencentes a famílias em situação de pobreza e de extrema pobreza, apresentaram agravos na saúde bucal quase duas vezes superiores à média regional, contrariando a hipótese que norteou este estudo.

\section{Agradecimentos}

Agradecemos à Secretaria de Assistência Social do município de Jaboatão dos Guararapes-PE, pela gentileza de ceder as listas das famílias cadastradas no Programa Bolsa Família, e aos agentes comunitários envolvidos nesta pesquisa.

Os autores agradecem ao Ministério da Educação (Capes) e ao Ministério de Ciência e Tecnologia (CNPq) pelo financiamento desta pesquisa.

\section{Abstract}

Objective: this study evaluated the impact of the Bolsa Familia program on the prevalence of dental caries in children 4 to 8 years old who received this benefit from birth. Methods: one hundred and sixty-three children were examined according to the criteria of the World Health Organization for decayed, missing, and filled teeth. Results: it was verified that the prevalence of dental caries of children included in the program was $78.5 \%$ and the average index of decayed, extracted, and filled teeth (dmft) in primary dentition was 4.77, with predominance of decayed teeth, which represents $81.8 \%$ of this ratio. The DMFT index (decayed, missing, and filled teeth in permanent dentition) found was 0.56 , indicating early disease in newly erupted permanent teeth. Conclusion: although this social program may promote immediate relief from the condition of extreme poverty, children born and raised with this benefit are still presenting oral health indicator of almost double the regional average.

Keywords: Child. Dental caries. Government programs.

\section{Referências}

1. Loretto NRM, Seixas ZA, Jardim MC, Brito RL. Cárie dentária no Brasil: alguns aspectos sociais, políticos e econômicos. Rev ABO Nac 2000; 8:45-9.

2. Jones CM, Worthington H. Water fluoridation, poverty and tooth decay in 12-year-old children. J Dent 2000; 28(6):38993.

3. Maltz M, Silva BB. Relação entre cárie, gengivite e fluorose e nível socioeconômico em escolares. Rev Saúde Pública 2001; 35(2):170-6. 
4. Martins MD, Araújo RGD, Veloso NF. Avaliação das necessidades de tratamento odontológico de crianças de baixa renda. J Bras Odontopediatr Odontol Bebê 1999; 2:132-6.

5. Spolidorio DMP, Hofling JF, Moreira D, Rodrigues JAO, Boriollo MFG, Rosa EAR. Dental caries status in deciduous and permanent dentition of Brazilian children aged 6-8 years with a socio-economic base. Braz J Oral Sci 2003; 2:147-50.

6. Brasil. Ministério da Saúde. Saúde no Brasil: contribuições para a Agenda de Prioridades de Pesquisa/Ministério da Saúde. Brasília: Ministério da Saúde; 2004. (Série B. Textos Básicos de Saúde)

7. Brasil. Ministério da Saúde. Departamento de Atenção Básica. Coordenação-Geral de Política de Alimentação e Nutrição. Manual de orientações sobre o Bolsa Família/Ministério da Saúde, Secretaria de Atenção à Saúde, Departamento de Atenção Básica, Coordenação-Geral de Política de Alimentação e Nutrição. Brasília: Ministério da Saúde; 2005.

8. Soares F, Ribas RP, Osório, RG. Evaluating the impact of Brazil's Bolsa Família: cash transfer programmes in comparative perspective. IPC Evaluation Note 2007; 1:1-11.

9. Brasil. Ministério da Saúde. Projeto SB Brasil 2010: condições de saúde bucal da população brasileira 2010 - resultados principais. Brasília: Ministério da Saúde; 2011.

10. Instituto Brasileiro de Geografia e Estatística (IBGE). PNAP. Pesquisa Nacional. Amostra de Domicílios [online] 2010 [citado 2012 Jul dia]. Disponível em URL: http://www.ibge.gov. br.

11. Brasil. Ministério do Desenvolvimento Social e Combate à Fome. Secretaria de Assistência Social, SIGPBF - Sistema de Gestão do Programa Bolsa Família [online] ano [citado 2012 Jul dia]. Disponível em URL: http://www.mds.gov.br/ bolsafamilia.

12. Hertzman C, Boyce T. How experience gets under the skin to create gradients in developmental health. Annu Rev Public Health 2010; 31:329-47.

13. Mota E, Carvalho DMT. Sistemas de informações em saúde. In: Rouquayrol MZ, Almeida Filho N. Epidemiologia \& saúde. 6. ed. Rio de Janeiro: MEDSI; 2003. p. 605-28.

14. Fejerskov O, Manji F. Risk assessment in dental caries. In: Bader JD. Risk assessment in dentistry. Chapel Hill, University of North Carolina Dental Ecology; 1990:215-7.

15. List L. Inequalities in dental attendance throughout the lifecourse. J Dent Res 2012; 91:91-7.

16. Attin T, Mbiydzemo FN, Villard I, Kielbassa AM, Hellwig E. Dental status of schoolchildren from a rural community in Cameroon. S Afr Dent J 1999; 54(4):145-8.

17. Carstens IL, Louw AJ, Kruger E. Dental status of rural school children in a sub-optimal fluoride area. J Dent Assoc S Afr 1995; 50(9):405-11.

18. Camelo RS, Tavares PA, Saiani CCS. Alimentação, nutrição e saúde em programas de transferência de renda: evidências para o Programa Bolsa Família. Revista Economia 2009; 10 (4):685-713.

19. Resende ACC. Avaliando resultados de um programa de transferência de renda: o impacto do Bolsa-Escola sobre os gastos das famílias brasileiras. [Dissertação Belo Horizonte]; Faculdade de Ciências Econômicas da Universidade Federal de Minas Gerais, Belo Horizonte 2006.

20. Associação Brasileira da Indústria de Higiene Pessoal, Perfumaria e Cosméticos (ABIHPEC). Classes D e E puxam consumo de produtos de higiene pessoal [online] 2010 [citado
2012 Jul dia]. Disponível em URL: http://http://www.abihpec. org.br/.

21. Hobdell MH, Myburgh NG, kelman M, Hausen H. Setting global goals for oral health for the year 2010. Int Dent J 2000; 50:245-9.

22. Barros AJD, Bertoldi AD. Desigualdades na utilização e no acesso a serviços odontológicos: uma avaliação em nível nacional. Cien Saude Colet 2002; 7(4):709-17.

23. Soares F, Soares S, Medeiros M, Osório R. Programas de transferência de renda no Brasil: impactos sobre a desigualdade. Brasília: IPEA; 2006. (Texto para Discussão, n. 1228).

\section{Endereço para correspondência:}

Valdeci Elias dos Santos Junior

Rua São Sebastião, 417/101

Piedade - 54410-500

Jaboatão dos Guararapes/PE

Fone: (081) 97446105

E-mail: valdeciodonto@gmail.com

Recebido: 06/03/2013. Aceito: 24/06/2013. 